\title{
Models of Return to Work for Musculoskeletal Disorders
}

\section{Izabela Z. Schultz • Anna W. Stowell •}

Michael Feuerstein • Robert J. Gatchel

Erratum to: Journal of Occupational Rehabilitation, DOI: 10.1007/s10926-007-9071-6

The following information was left out:

For Fig 1. underneath the text:

With kind permission of Springer Science and Business Media.

For Fig. 2. underneath the text:

With kind permission of Springer Science and Business Media.

For Fig. 3. underneath the text:

Reprinted with permission from the National Academies Press, Copyright 2001, National Academy of Sciences. 